\title{
Low doses effect in GaP light-emitting diodes
}

\author{
O.M. Hontaruk ${ }^{1}$, O.V. Konoreva ${ }^{1}$, Ye.V. Malyi ${ }^{1}$, I.V. Petrenko ${ }^{1}$, M.B. Pinkovska ${ }^{1}$, \\ O.I. Radkevych ${ }^{2}$, V.P. Tartachnyk ${ }^{1}$ \\ ${ }^{I}$ Institute for Nuclear Researches, NAS of Ukraine, 47, prospect Nauky, 03028 Kyiv, Ukraine \\ ${ }^{2}$ SE “RI of Microdevices” STC “Institute for Single Crystals”, NAS of Ukraine, \\ 3, Pivnichno-Syretska str., 04136 Kyiv, Ukraine \\ Corresponding author phone: +38(044)-525-37-49; e-mail: evgen.malyj@gmail.com
}

\begin{abstract}
The paper is devoted to the electrophysical characteristics study of serial red and green GaP light-emitting diodes (LEDs) irradiated with low $\alpha$-particles doses $(\Phi \leq$ $10^{12} \mathrm{~cm}^{-2}$ ). It was stated that radiation features of $p-n$-junction and its capacitance change in dependence on temperature. The capacitance grows at $300 \mathrm{~K}$, and drops at $77 \mathrm{~K}$. At the same time, a direct branch of current-voltage characteristics shifts into the lower voltage direction, and appropriative barrier potential reduction value from 6.5 down to $3.5 \mathrm{eV}$ is observed. The effects are caused by radiation defects, charge state of which depends on the Fermi level in GaP. The assumption has been made about high ionization level role in the sulfur impurity transition process into electrically active donor position.
\end{abstract}

Keywords: gallium phosphide, GaP, LEDs, CVC, $\alpha$-particles, irradiation, low doses, capacitance.

Manuscript received 14.12.15; revised version received 05.04.16; accepted for publication 08.06.16; published online 06.07.16.

\section{Introduction}

It is known that radiation defects induced by fast particles in light-emitting diodes (LEDs) causes appearing of deep energy levels in semiconductor band gap. They capture charge carriers and affect the $p$ - $n$ junction parameters. Irradiation leads to $p$ - $n$-junction capacitance reduction, the differential resistance growth, potential barrier value decrease among semiconductor regions with different conductivity types and base resistance increase.

However, this situation is not always realized in practice. Despite numbers of publications devoted to this problem [1-6], one can hardly consider sufficient information amount needed to predict behavior of the devices in extreme conditions. This is especially true concerning the early irradiation stages, the so-called "low doses effect" that is observed at crystal electron subsystem excitation high levels.

In this paper, the results of the $\alpha$-particles effect researches on the electrical gallium phosphide LEDs characteristics and diode behavior peculiarities at initial irradiation stages are presented. It should be also noted that one can use "positive" effect observed at low doses to correct performances of the industrial diode structures. 


\section{Experimental}

Measurements were carried out using the red and green LEDs obtained by double liquid epitaxy of a solutionmelt limited volume on the substrate (111) grown by Czochralski method. The substrate thickness was approximately $200 \mu \mathrm{m}$; the epitaxial films thicknesses were $d_{n}=50 \ldots 60 \mu \mathrm{m}, d_{p}=20 \ldots 30 \mu \mathrm{m}$. The electron conductivity of substrate film and $n$-type film were obtained using Te doping; the hole conductivity of $p$ type film was reached using $\mathrm{Zn}$. Nitrogen was green emission activator, and $\mathrm{Zn}-\mathrm{O}$ complex was red emission activator. The $p$-doped region level was nearly 5 times higher than the main dopant concentration in $p-n-$ junction $n$-region. Current-voltage (CVC) and capacitance-voltage characteristics of the diodes were measured at 77 and $300 \mathrm{~K}$. Samples were irradiated with $\alpha$-particles in U-120 cyclotron at room temperature, $E_{\alpha}=$ $27 \mathrm{MeV}$; fluence was $\Phi \leq 10^{12} \mathrm{~cm}^{-2}$.

It is possible to estimate the $\alpha$-particle penetration depth $R_{\alpha}$ with the energy $E$ in GaP crystal by using the known ratio:

$$
R_{\alpha}=10^{-4} E^{\frac{3}{2}} \frac{\sqrt{A}}{\rho},
$$

where $E$ is a particle's energy, $\rho$ - material density, $A-$ substance atomic weight.

Since $\mathrm{GaP}$ is a binary compound, evaluation of the penetration depth can be done in two ways based on the average $\mathrm{GaP}$ atomic weight $A \approx 50$ or separately for $\mathrm{Ga}$ and $\mathrm{P}$ atoms, and then by determination of their average. The first method gives the value $R_{\alpha}=253 \mu \mathrm{m}$; the second is $292 \mu \mathrm{m}$; the values are quite close to each other. The total sample thickness was $d \leq 290 \mu \mathrm{m}$. Therefore, for given small epitaxial films thickness one can consider that radiation defects are uniformly distributed inside the $p-n$-junction.

\section{Results and discussion}

Applying an external voltage to the diode structure leads to changes in the space charge value, which manifests itself in a changing capacitance. It is known that irradiation of $\mathrm{GaP}$ diodes with fast electrons $(E=1 \mathrm{MeV})$ in moderate and high doses leads to a decrease in the capacitance of $p-n$-junction [7] due to deep levels of radiation defects. The main reason for the destructive radiation effects is entering the deep levels of radiation defects. The concentration of major carriers reduces, and barrier capacitance dose dependent of the integrated irradiation flow $C(\Phi)$, when they capture the charge carriers for abrupt $p$ - $n$-junction, is well described by the expression [8]:

$$
C(\hat{O})=S \sqrt{\frac{\varepsilon \varepsilon_{0} q N_{D}}{2\left(U+U_{\tilde{N}}\right)}} e^{-K_{n} \frac{\hat{O}}{2}},
$$

where $N_{D}$ is the concentration of initial donors in poorly doped $p$ - $n$-junction part, $U$ - reverse voltage, $U_{C}-$ difference of contact potentials, $K_{n}$ - relative carriers' removal rate during irradiation or radiation damageability $\left(K_{n}=\frac{1}{n_{0}} \cdot \frac{d n}{d \Phi}\right), S$ - junction area.

This dependence $C(\Phi)$ is observed for a certain range of other irradiation types, including $\alpha$-particles, if to take into account the specific radiation damageability coefficient $K$. As for low $\alpha$-particles doses $\left(\Phi \leq 10^{12} \mathrm{~cm}^{-2}\right)$, the opposite effect was observed in the experiment: the irradiated $p$ - $n$-junction capacitance measured at $T=300 \mathrm{~K}$ is higher than the initial sample capacitance; while at low temperatures the sample capacitance drops (Fig. 1). At the same doses, the shift of direct branches in current-voltage characteristics toward lower voltages occurs, and appropriative potential barrier value drops from 6.5 down to $3.5 \mathrm{eV}$ (Fig. 2).
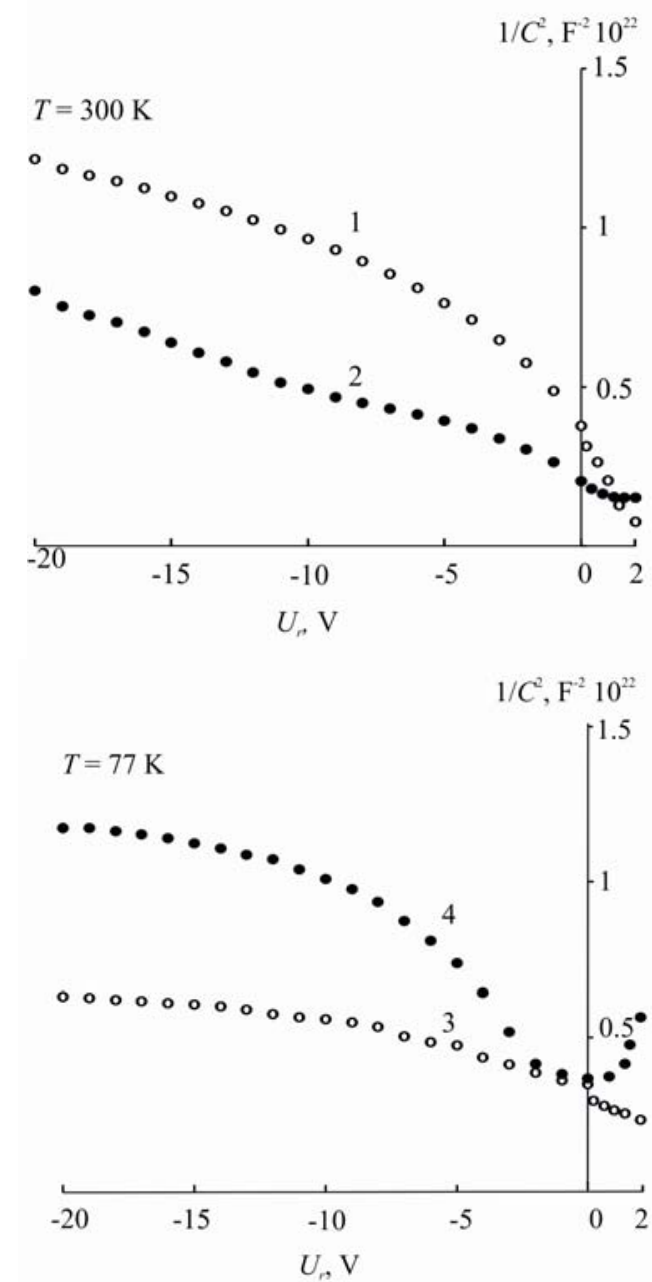

Fig. 1. Capacitance-voltage characteristics of initial and $\alpha$ irradiated GaP:N LEDs measured at 300 and $77 \mathrm{~K}: 1,3-$ initial samples; 2,4 - irradiated with $\Phi=10^{12} \alpha$-particles $/ \mathrm{cm}^{2}$. 


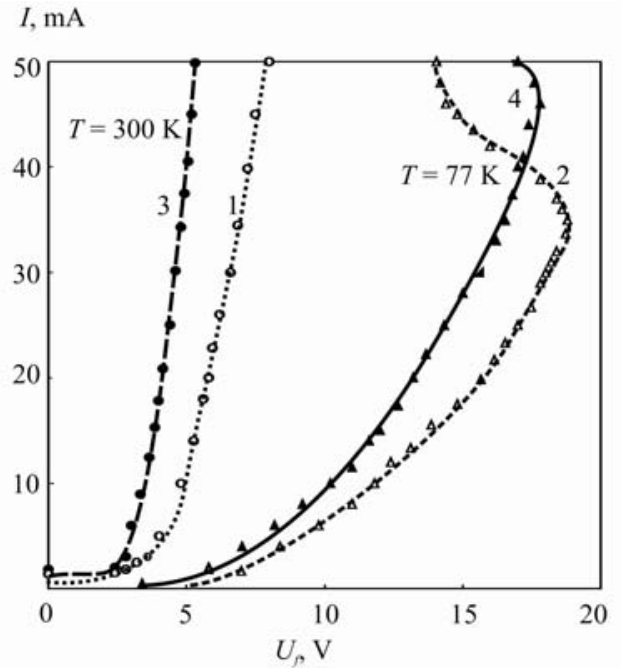

Fig. 2. Current-voltage characteristics of initial $(1,2)$ and $\alpha$ irradiated $(3,4)$ with $\Phi=10^{12} \mathrm{~cm}^{-2}, E=26 \mathrm{MeV}$ GaP:Zn,O LEDs measured at 300 and $77 \mathrm{~K}$.

Opposite changes in the irradiated $p$ - $n$-junction capacitance measured at 77 and $300 \mathrm{~K}$ indicate the dependence of the defects charge state introduced by $\alpha$ particles relatively to the Fermi level $E_{\mathrm{F}}$ position in semiconductor. It is possible to obtain temperature dependence of $E_{\mathrm{F}}$, being based on the known ionization energy values of major donors and acceptors (Te and $\mathrm{Zn}$ ) in $\mathrm{GaP}$.

The carrier charge concentration dependences on temperature for $n$ - and $p$-types of materials are given in Fig. 3. In the former case, the conductivity is determined by one level $\left(E_{d}=0.14 \mathrm{eV}\right)$, and in the latter one by two nearly lying activation energies $\left(E_{a 1}=0.071 \mathrm{eV}, E_{a 2}=\right.$ $0.04 \mathrm{eV})$. The difference in the donor ionization energy values $E_{d}=0.14 \mathrm{eV}$ and $E_{d}^{\mathrm{Te}}=0.09 \mathrm{eV}$ is apparently caused by a high compensation level of the studied crystal conductivity [9].

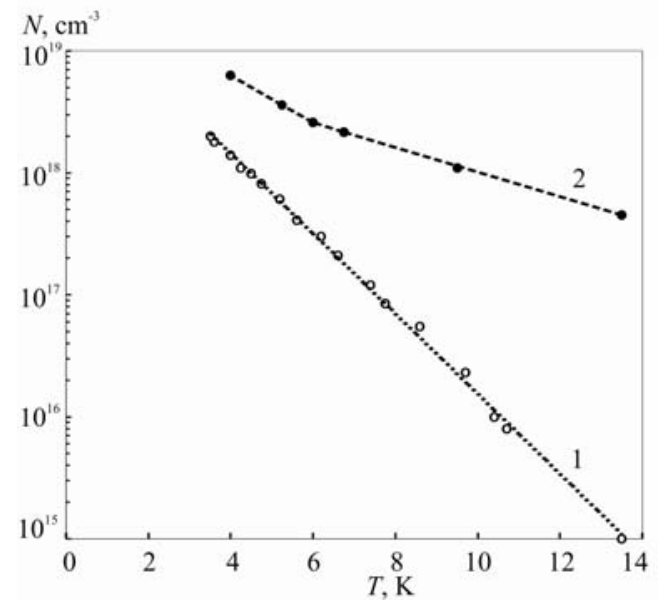

Fig. 3. Dependences of the charge carrier concentration on temperature for: $1-n$-type conductivity $\left(E_{d}=0.14 \mathrm{eV}\right)$ and $2-$ $p$-type conductivity $\left(E_{a 1}=0.071 \mathrm{eV}\right.$ and $\left.E_{a 2}=0.04 \mathrm{eV}\right) \mathrm{GaP}$ crystals.
The average acceptor activation value energy is close to $E_{a}^{\mathrm{Zn}}=0.06 \mathrm{eV}$. To assess the relationship between the position of $E_{\mathrm{F}}$ on temperature, we used activation energies values $E_{d}^{\mathrm{Te}}=0.09 \mathrm{eV}$ and $E_{a}^{\mathrm{Zn}}=$ $0.06 \mathrm{eV}$, being based on the calculation methods proposed in [10].

At low temperatures, when ionized donor impurity dominated in changing the carrier concentration $\left(p<<N_{D}^{+}\right)$, the neutrality equation simplifies and takes the form [10]:

$$
n=N_{C} e^{-\frac{E_{C}-E_{\mathrm{F}}}{k T}}=p_{D}=\frac{N_{D}}{2 e^{\frac{E_{\mathrm{F}}-E_{d}}{k T}}+1},
$$

where $p_{D}$ is the hole concentration on donors.

By denoting $e^{\frac{E_{\mathrm{F}}}{k T}}=\chi$, we get a quadratic equation, solution of which is as follows:

$\chi=\frac{1}{4} e^{\frac{E_{D}}{k T}}\left(\sqrt{1+\frac{8 N_{D}}{N_{C}} \cdot e^{\frac{\Delta E_{D}}{k T}}}-1\right)$.

Then

$E_{\mathrm{F}}=k T \ln \frac{1}{4} e^{\frac{E_{D}}{k T}}\left(\sqrt{1+\frac{8 N_{D}}{N_{C}} \cdot e^{\frac{\Delta E_{D}}{k T}}}-1\right)$

where $\Delta E_{D}$ is the energy of impurity ionization.

At low temperatures:

$$
\begin{aligned}
& A=\frac{8 N_{D}}{N_{C}} \cdot e^{\frac{\Delta E_{D}}{k T}}>>1, \\
& E_{\mathrm{F}}=\frac{E_{C}+E_{D}}{2}+\frac{k T}{2} \ln \frac{N_{D}}{2 N_{C}} .
\end{aligned}
$$

Let us estimate the value $A$ in $\mathrm{GaP}$ for two temperatures: 77 and $300 \mathrm{~K}$. Considering $N_{C, \mathrm{GaP}}^{77}=6.75 \cdot 10^{23} \mathrm{~m}^{-3}, N_{C, \mathrm{GaP}}^{300 \mathrm{~K}}=5.2 \cdot 10^{24} \mathrm{~m}^{-3}, \Delta E_{D}=$ $0.09 \mathrm{eV}$, obtain $A_{\mathrm{GaP}}^{77 \mathrm{~K}}=990795, \quad A_{\mathrm{GaP}}^{300 \mathrm{~K}} \cong 5$. So, to obtain $E_{\mathrm{F}}(T)$ dependence at temperatures close to the room one, it is necessary to use the exact ratio (5), at low temperatures - expression (7).

The calculation results of $E_{\mathrm{F}}(T)$ for $n$-type gallium phosphide are shown in Fig. 4 . We see that at $T=77 \mathrm{~K}$ the Fermi level is situated $0.05 \mathrm{eV}$ below the conduction band bottom; temperature increase shifts the $E_{\mathrm{F}}$ position towards the forbidden gap middle, and at $T=300 \mathrm{~K} E_{\mathrm{F}}$ is at the distance greater than $0.1 \mathrm{eV}$ from the bottom of the conduction band $E_{C}$. The donor level of $\sim 0.1 \mathrm{eV}$ depth at $T=77 \mathrm{~K}$ is fully filled in and can not affect the barrier capacitance value. When approaching to room temperature, recharging the level occurs. It ionizes and begins to affect the junction capacitance by increasing it. 


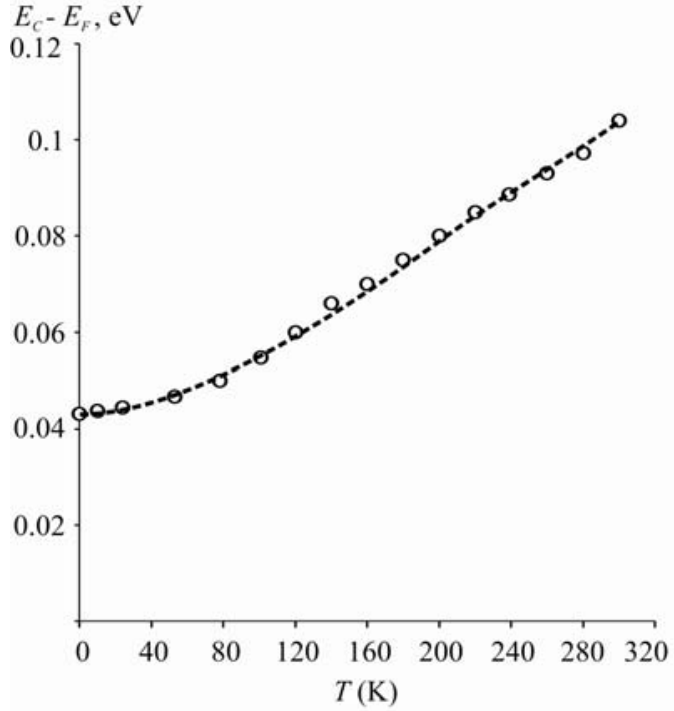

Fig. 4. Dependence of the Fermi level position on temperature in $n$-type GaP, $n=10^{17} \mathrm{~cm}^{-3}$.

Sulfur impurity with the ionization energy $E_{d}=$ $0.104 \mathrm{eV}$ [9] as uncontrolled and introduced during irradiation might be such center. Indeed, bombardment of the crystal $\mathrm{GaP}$ with $\alpha$-particles $(E=27.2 \mathrm{MeV})$ causes reactions in the ${ }_{31}^{15} \mathrm{P}$ and ${ }_{69}^{31} \mathrm{Ga}$ nuclei, as the $\alpha$ particle energy is much higher than penetration threshold for both mentioned nuclei $E_{b}^{\mathrm{P}}=9.55 \mathrm{MeV}-$ phosphorous and $E_{b}^{\mathrm{Ga}}=13.33 \mathrm{MeV}$ - gallium.

${ }_{31}^{15} \mathrm{P}+\alpha^{++} \rightarrow{ }_{34}^{17} \mathrm{Cl}+n_{0}$,

${ }_{34}^{17} \mathrm{Cl} \underset{32.2 \min }{\stackrel{\beta^{+}}{\rightarrow}}{ }_{34}^{16} \mathrm{~S}_{\text {stable }}$

${ }_{69}^{31} \mathrm{Ga}+\alpha^{++} \rightarrow{ }_{73}^{33} \mathrm{As}+\gamma, T^{\frac{1}{2}}=90$ days,

${ }_{71}^{31} \mathrm{Ga}+\alpha^{++} \rightarrow{ }_{75}^{33} \mathrm{As}_{\text {stable }}$.

Prevalence of ${ }_{31}^{15} \mathrm{P}$ isotope is $100 \%,{ }_{69}^{31} \mathrm{Ga}$, ${ }_{71}^{31} \mathrm{Ga}$ isotopes are 60.4 and $39.6 \%$, respectively $[11,12]$. As it is obvious from the daughter products scheme, in all three cases gallium phosphide is doped by donors, including sulfur. This is fully consistent with previously made assumptions about the donor's role with the $0.1 \mathrm{eV}$ depth in the increasing capacitance process of irradiated $p$ - $n$-junction at $T=300 \mathrm{~K}$. In the junction $p$-region, this effect should be opposite, but it is nearly invisible because of a much higher doping level, and thus its influence on the $p$ - $n$-transition capacitance is much weaker.

To ensure the correctness of such considerations, let us estimate the quantitative contribution of the inelastic collisions of $\alpha$-particles with Ga and P nuclei. It is small. The cross sections of these processes are determined by a nuclear interaction radius, which is about one Fermi $\left(10^{-13} \mathrm{~cm}\right)$ and for an intermediate $\alpha$ particle energy (including $E_{\alpha}=27.2 \mathrm{MeV}$ ) are equal to $1 \ldots 100$ mbarn. Since $\alpha$-particle interaction with nucleus is single-stage, no need to consider repeated interaction acts and cascading function value, one can use the expression for the number of primary inelastic collisions $\alpha \rightarrow \mathrm{P}$ as:

$N_{S}=N_{a} \cdot \sigma \cdot \Phi$

where $N_{a}$ is the concentration of phosphorous atoms, $\sigma-$ $\alpha$-particles interaction cross section with $\mathrm{P}$ atoms.

The estimated value of sulfur atoms' number $N_{S} \approx 10^{9}$ is low as compared with the concentration of atoms in the $\mathrm{GaP}$ crystal. By analyzing the irradiation effects on the investigated structure, we must also take into account the interaction specificity of the heavy doubly charged particle with crystal atoms. Comparison of line energy loss for a heavy charged particle on atom displacement and ionization gives:

$\frac{\left(\frac{d E}{d x}\right)_{\text {ion }}}{\left(\frac{d E}{d x}\right)_{d i s}} \cong 10^{3}$.

So, most of the $\alpha$-particles energy is spent on ionization.

It was shown [6] that in polar semiconductors, as well as in irradiated with low doses GaP, the role of getering the radiation-stimulated defects is increased, which results in increasing the minority carriers' lifetime, diffusion length and others. This effect is strengthen by recombination-accelerated diffusion mechanisms that can effectively manifest itself in widegap semiconductors [12].

Therefore, in the wide-zone GaP at high ionization levels simultaneously with $\mathrm{S}$ donor's concentration increasing due to nuclear reactions, the process of getering can occur - the $p$ - $n$-transition clearing out of impurities in metastable state and active replacing process by the Watkins model [13].

It is clear that the capacitance changes in the red and green LEDs observed at low $\alpha$-particle doses occur due to all the mentioned factors, and sulfur atoms transition to electrically active donor state dominates, if the ionization level is sufficiently high.

\section{Conclusion}

It is shown that the low dose effect appearing in $\mathrm{GaP}$ LEDs irradiated with $\alpha$-particles $\left(\Phi=2 \cdot 10^{12} \mathrm{~cm}^{-2}\right)$, which manifests itself as "improvement" of parameters: the junction capacitance increasing at room temperature, decreasing in the potential barrier between $p$ - $n$-regions and the differential resistance value in CVC. These features are caused by $\alpha$-particle nuclear reactions and high ionization levels that give rise to additional donors. 
The nuclear reactions product: donors $\mathrm{S}$ and interstitial atoms $\mathrm{S}$, which can substitute phosphorous atoms at high ionization level and are responsible for the effect. At high excitation of the crystal electron subsystem significant contribution to the structural ordering of the transition region provides the effect of radiationstimulated defects' getering.

\section{References}

1. V.S. Vavilov, A.N. Kiv, O.R. Niyazova, Formation and Migration Mechanism of the Defects in Semiconductors. Nauka, Moscow, 1981, p. 368 (in Russian).

2. V.A. Vinetskii, G.A. Holodar, Radiation Semiconductors Physics. Kyiv, Naukova Dumka, 1979, p. 335 (in Russian).

3. O.Yu. Borkovskaja, N.L. Dmitruk, V.G. Litovchenko, O.N. Mishchuk, To model of the radiationstimulated ordering effect in semiconductors $\mathrm{A}^{3} \mathrm{~B}^{5}$ // Semiconductors, 23(2), p. 207-212 (1989), in Russian

4. O.Yu. Borkovskaya, N.L. Dmitruk, R.V. Konakova, V.G. Litovchenko, Yu.A. Tkhorik, V.I. Shakhovtsov, Effect of the interface on radiation defect formation in gallium arsenide // Radiation Effects and Defects in Solids, 42 (3-4), p. 249-251 (1979).

5. O.Yu. Borkovskaja, N.L. Dmitruk, R.V. Konakova, V.G. Litovchenko, V.I. Shakhovtsov, Influence of $\gamma$ - and electron irradiation on recombination characteristics near the surface of GaAs // phys. status solidi (a), 58 (1), p. k25-k28 (1980).

6. O.Yu. Borkovskaja, N.L. Dmitruk, R.V. Konakova, V.G. Litovchenko Radiation effects at the gallium phosphide boundary zone // Zhurnal tekhnicheskoi fiziki, 52 (6), c. 1194-1199 (1982), in Russian

7. V.P. Tartachnyk, Radiation defects in the semiconductors phosphides $\mathrm{A}^{3} \mathrm{~B}^{5}$ and $\mathrm{A}^{2} \mathrm{~B}_{2}^{5} / /$ Thesis summary on Doctor of Science, Nauka, Kyiv, 1993, p. 30 (in Ukrainian).

8. F.P. Korshunov, G.V. Gatalskii, G.M. Ivanov, Radiation Effects in Semiconductor Devices. Nauka i tekhnika, Minsk, 1978, p. 230 (in Russian).

9. V.M. Andreev, L.M. Lolginov, D.N. Tretyakov, Liquid Epitaxy in Semiconductor Instruments Technology. Sovetskoe radio, Moscow, 1975, p. 328 (in Russian),

10. P.S. Kireev, Semiconductor Physics. Vysshaya shkola, Moscow, 1969, p. 590 (in Russian).

11. B.S. Dzhelepov, L.P. Pekar, Radioactive Nuclei Decay Schemes. Nauka, Moscow, 1966, p. 750 (in Russian).

12. L.S. Smirnov, Semiconductors Doping by Nuclear Reactions Method. Nauka, Novosibirsk, p. 180 (1981) in Russian.

13. M.A. Elango, Elementary Inelastic Radiation Processes. Nauka, Moscow, 1988, p. 150 (in Russian). 